\title{
ANKLE
}

\section{A 3-portal approach for arthroscopic subtalar arthrodesis}

\author{
Lijkele Beimers · Peter A. J. de Leeuw • \\ C. Niek van Dijk
}

Received: 6 February 2009/ Accepted: 25 March 2009/Published online: 17 April 2009

(c) The Author(s) 2009. This article is published with open access at Springerlink.com

\begin{abstract}
We present a 3-portal approach for arthroscopic subtalar arthrodesis with the patient in the prone position. The prone position allows the use of the two standard posterior portals and it allows for accurate control of hindfoot alignment during surgery. Furthermore, the introduction of talocalcaneal lag screws is easy with the patient in this position. In addition to the standard posterior portals, an accessory third portal is created at the level of the sinus tarsi for introduction of a large diameter blunt trocar to open up the subtalar joint. Due to the curved geometry of the posterior subtalar joint, removal of the anterior articular cartilage is impossible by means of the posterior portals only. An advantage of the 3-portal approach is that ring curettes can be introduced through the accessory sinus tarsi portal to remove the articular cartilage of the anterior part of the posterior talocalcaneal joint. Arthroscopic subtalar arthrodesis in patients with a talocalcaneal coalition presents a technical challenge as the subtalar joint space is limited. The 3-portal technique was successfully used in three subsequent patients with a talocalcaneal coalition; bony union of the subtalar arthrodesis occurred at 6 weeks following surgery. With the 3-portal technique, a safe and time-efficient arthroscopic subtalar arthrodesis can be performed even in cases with limited subtalar joint space such as in symptomatic talocalcaneal coalition.
\end{abstract}

L. Beimers $(\bowtie)$ P. A. J. de Leeuw · C. N. van Dijk

Department of Orthopedic Surgery, Academic Medical Centre,

University of Amsterdam, Meibergdreef 9,

1105 AZ Amsterdam, The Netherlands

e-mail: 1.beimers@amc.uva.nl
Keywords Subtalar joint · Arthroscopic - Arthrodesis · Talocalcaneal coalition

\section{Introduction}

In 2000, a 2-portal posterior approach for hindfoot arthroscopy with the patient in the prone position was introduced [12]. This approach was successfully used for arthroscopic subtalar arthrodesis in a series of patients with post-traumatic osteoarthritis [6]. A painful talocalcaneal coalition is a recognized indication for talocalcaneal arthrodesis in skeletally mature patients [8,9]. The presence of a talocalcaneal coalition presents a technical challenge since the bar only allows limited opening up of the joint during surgery. As standard arthroscopic techniques for subtalar arthrodesis do not provide means of opening up the joint, they are difficult to use in patients with limited subtalar joint space. An accessory posterolateral portal for introduction of a blunt trocar for subtalar joint distraction in arthroscopic subtalar joint arthrodesis was described [1,4]. However, the working space in the hindfoot is significantly reduced by using three posterior portals in the hindfoot. We present a technique for arthroscopic subtalar arthrodesis based on the 2-portal posterior approach with the patient in the prone position. Via an accessory third working portal at the level of the sinus tarsi, a large diameter blunt trocar is introduced in order to provide subtalar joint opening. The sinus tarsi portal is also used for introduction of ring curettes in order to remove the cartilage of the anterior part of the posterior talocalcaneal joint. We describe the technique and the results of three subsequent patients with a symptomatic talocalcaneal coalition who successfully underwent arthroscopic posterior subtalar arthrodesis using this 3portal approach. 


\section{Operative technique}

The patient is placed in the prone position. A tourniquet is inflated around the thigh. A triangular-shaped pad is placed under the lower leg to provide unconstrained motion of the ankle joint during surgery (Fig. 1). A lateral support is placed against the ipsilateral hip and the operating table is tilted towards the ipsilateral side. Following prepping and draping, the Achilles tendon and the lateral and medial malleolus are identified. The two standard posterior portals for hindfoot endoscopy are created using the technique as described before [12]. First the posterolateral portal is made at the level or slightly above the tip of the lateral malleolus, just lateral to the Achilles tendon with the foot in the neutral position (Fig. 1). After making a longitudinal skin incision, the subcutaneous layer is split using a mosquito clamp. The mosquito clamp is pointed anteriorly, in the direction of the interdigital webspace between the first and second toe. When the tip of the clamp touches bone, it is exchanged for a $4.5 \mathrm{~mm}$ arthroscope shaft with the blunt trocar pointing in the same direction. By palpating the bone in the sagittal plane, the level of the ankle joint and subtalar joint most often can be identified because the prominent
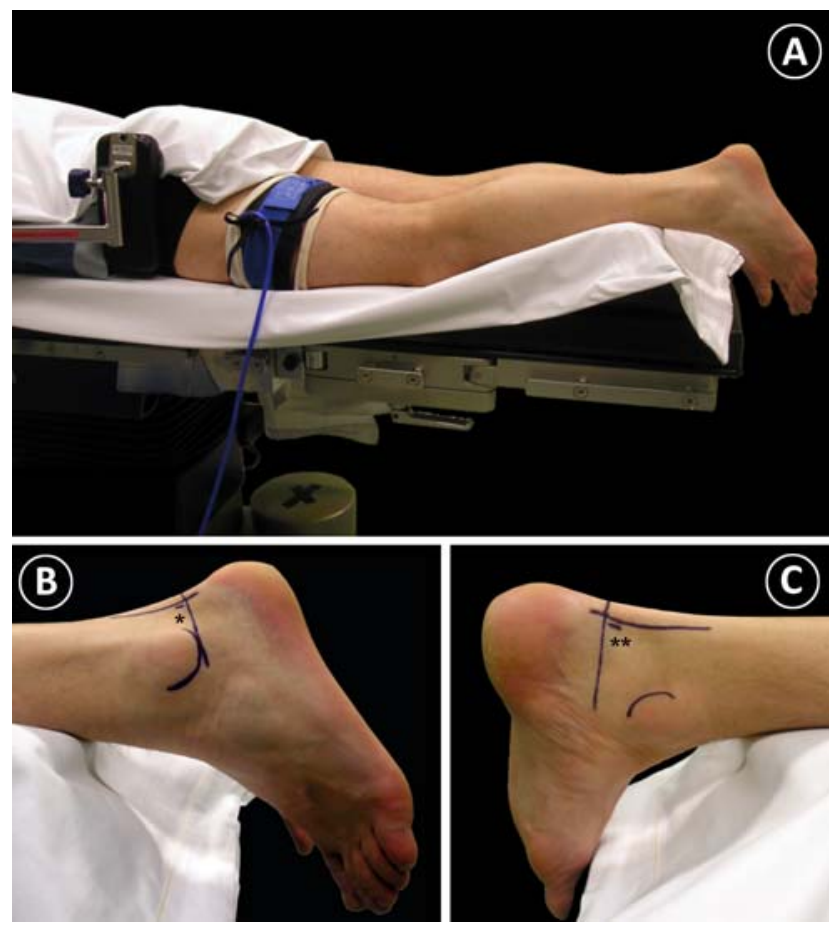

Fig. 1 a Patient positioning for arthroscopic subtalar arthrodesis. A triangular-shaped padding supports the lower leg for unconstrained ankle joint motion. A tourniquet is applied to the thigh. Note the lateral support for safe tilting of the patient to the ipsilateral side. b The posterolateral portal is made at the level or slightly above the tip of the lateral malleolus, just lateral to the Achilles tendon. c The posteromedial portal is made just medial to the Achilles tendon at the same height as the posterolateral portal posterior talar process can be felt in between the joints. The blunt trocar remains extra-articular at the level of the ankle joint. Subsequently, the posteromedial portal is created just medial to the Achilles tendon at the same level as the posterolateral portal (Fig. 1). After making the longitudinal skin incision, a mosquito clamp is introduced and directed straight towards the arthroscope shaft. When the mosquito clamp touches the shaft, it is used as a guide to move the mosquito clamp over the shaft towards the ankle joint. All the way down to the ankle joint, the tip of the mosquito clamp has to touch the arthroscope shaft until the tip touches the bone. The blunt trocar is then exchanged for a $30^{\circ}$ $4.0 \mathrm{~mm}$ arthroscope. The arthroscope is slightly pulled back until the tip of the mosquito clamp comes into view. The clamp is used to spread the extra-articular soft tissues in front of the tip of the arthroscope. In case of scar tissue or adhesives, the mosquito clamp is exchanged for a $5.0 \mathrm{~mm}$ full-radius shaver. The fatty tissue overlying the capsule of the subtalar joint is removed. After removal of the joint capsule of the subtalar joint, the posterior compartment of the subtalar joint is visualized. The posterior talar process can be freed from scar tissue and the flexor hallucis longus (FHL) tendon is identified medially. The FHL tendon is an important anatomic landmark in hindfoot endoscopy, as the posteromedial neurovascular bundle is located medially from the FHL tendon. Release of the flexor retinaculum from the posterior talar process is performed to have better access to the subtalar joint. Via the posterior portals the cartilage of the posterior facet of the subtalar joint is now removed using ring curettes. In case of a talocalcaneal coalition, the working area in the posterior subtalar joint is restricted due to the bar between the talus and calcaneus. A talocalcaneal coalition is a congenital osteofibrous, cartilaginous, or osseous union of the talus and calcaneus. A talocalcaneal coalition ossifies either completely or incompletely between 12 and 16 years of age [10]. To open up the posterior subtalar joint, an accessory sinus tarsi portal is created for introduction of a large diameter blunt trocar. A small skin incision is made at the level of the sinus tarsi. (Fig. 2). A spinal needle is introduced via the sinus tarsi portal and is directed towards the tip of the lateral malleolus. At the level of the subtalar joint the spinal needle is pointing posteriorly. The arthroscope is used to check the position of the needle. Following removal of the spinal needle, the large diameter blunt trocar $(4.0 \mathrm{~mm})$ is inserted through the sinus tarsi portal and is manoeuvred towards the posterior subtalar joint. For the purpose of opening up the joint, the blunt trocar is now forced into the subtalar joint. Since the direction of the blunt trocar is almost parallel to the subtalar joint space it can be forced in a sidewards direction into the joint from laterally. The sideward movement prevents the trocar of making a false route into the subchondral bone (Fig. 3a). In 


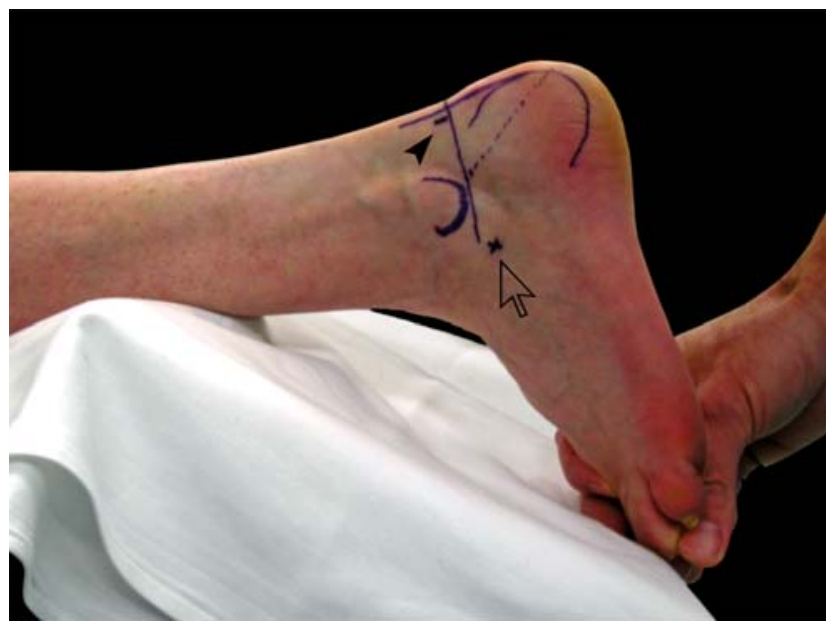

Fig. 2 The accessory portal for arthroscopic subtalar arthrodesis is located at the level of the sinus tarsi (arrow). The posterolateral portal is marked with a black arrow

case of a talocalcaneal coalition, the talus and calcaneus are connected by the talocalcaneal bar that is located at the medial side. A small size chisel $(4.0$ or $6.0 \mathrm{~mm})$ is placed through the posteromedial or posterolateral portal into the area of the bar. An attempt can be made to remove the bar by using the small size chisel in order to further open up the joint (Fig. 3b). Removal of the articular cartilage of the posterior subtalar joint using ring curettes is performed by changing portals (Fig. 3c-e). After removal of the articular cartilage, the subchondral bone is entered to expose the highly vascular cancellous bone. Using the small size chisel, a number of approximately $2.0 \mathrm{~mm}$ deep longitudinal grooves are made in the subchondral cancellous bone of the talus and calcaneus (Fig. 3f). A vertical skin incision is made at the tip of the heel for introduction of two lag screws. Using fluoroscopy, the $6.5 \mathrm{~mm}$ lag screws are placed across the posterior subtalar joint. The estimated length and direction of the two screws can be preoperatively planned on the lateral weightbearing radiograph of the ankle. Before insertion of the two screws it is important to check the alignment of the hindfoot. Coaptation of the posterior subtalar joint surfaces can be checked arthroscopically when tightening the screws (Fig. 3g). The skin is closed using non-resorbable sutures. A non-weightbearing lower leg cast is provided for 4 weeks, followed by a walker boot for another 2 weeks. At 6 weeks following surgery, anteroposterior and lateral weightbearing ankle radiographs are made. With radiographic signs of union of the subtalar arthrodesis, the patient is allowed full weightbearing without further support. For patient comfort, the walker boot can be applied for another 2 weeks.

\section{Patients}

From March 2006 to July 2006, three subsequent female patients with a painful talocalcaneal coalition (two left

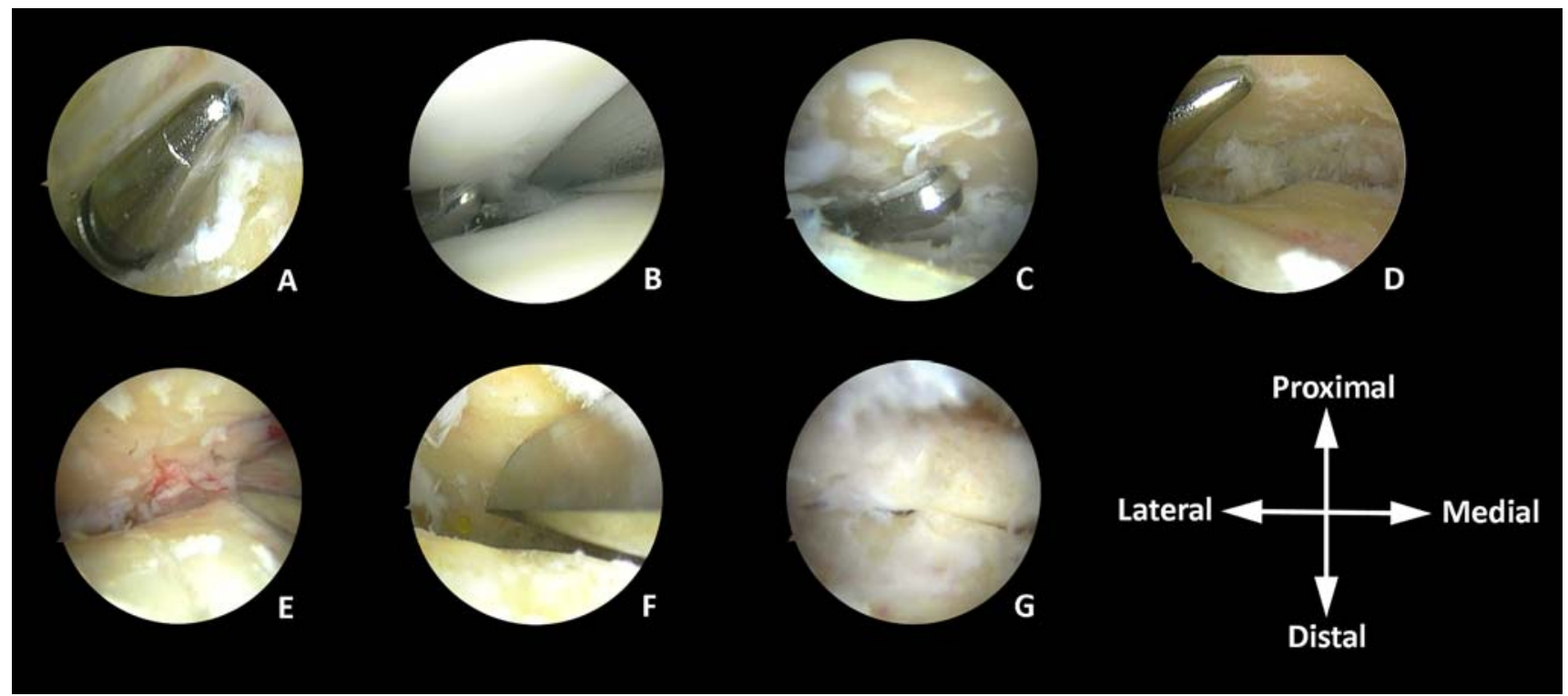

Fig. 3 Intra-operative views of arthroscopic subtalar arthrodesis in a patient with a talocalcaneal coalition. a The blunt trocar is positioned laterally of the subtalar joint via the accessory sinus tarsi portal. b The blunt trocar is sidewards forced into the subtalar joint. Using a small size chisel, an attempt is made to destruct the medially located talocalcaneal bar. c and d Ring curettes are used for removal of articular cartilage from the posterior subtalar joint. e It is important to remove all cartilage from the posterior subtalar joint. A bone cutter shaver may also be used for this purpose. f Longitudinal grooves are cut in the subchondral bone of the talus and calcaneus using the small size chisel. g Under arthroscopic view, the screws are tightened and coaptation of the posterior subtalar joint surfaces is seen 
and one right foot) were operated on by the senior author using the technique as described. Computed tomography (CT) scanning of the hindfoot confirmed the presence of a medially located coalition between the talus and calcaneus. (Fig. 4). As conservative treatment eventually failed, the decision was made to perform an arthroscopic isolated subtalar arthrodesis using the 3-portal technique as described. Resection of the talocalcaneal coalition was not considered since the patients were skeletally mature and the coalition was of larger extent [5, 9, 10]. In all patients joint opening and distraction using the large diameter blunt trocar introduced via the sinus tarsi portal, was sufficient to create enough working space to remove the articular cartilage from the posterior subtalar joint surfaces. The duration of surgery was on average $60 \mathrm{~min}$ (range, 52-65 min). Patients were discharged from the hospital the day after surgery. Post-operative radiographs 6 weeks following surgery, showed bony union of the subtalar arthrodesis in all three patients (Fig. 5). At time of follow-up (range, 24-28 months), none of the patients had any complaints with ambulation and all were satisfied with the results. No complications had occurred.

\section{Discussion}

Using the 3-portal hindfoot approach with the patient in the prone position, arthroscopically assisted arthrodesis of the posterior subtalar joint was successfully performed in three patients with a symptomatic talocalcaneal coalition. The talocalcaneal bar consists of osteofibrous tissue which allows for some micro-motion in the bar and the subtalar joint, thereby producing pain in the hindfoot on walking. Arthroscopic subtalar arthrodesis is technically challenging in patients with a talocalcaneal coalition since the bar is restricting access to the joint during surgery. The addition of an accessory portal at the level of the sinus tarsi provides safe access for introduction of a large diameter blunt trocar in order to open up the subtalar joint. This limited joint distraction is sufficient to allow introduction of ring curettes into the posterior subtalar joint. Due to the curvature of the posterior talocalcaneal joint it is not possible to remove the articular cartilage from the anterior part of the posterior talocalcaneal joint through the posterior portals. This will only be possible in case the talocalcaneal joint can be sufficiently opened. With a talocalcaneal bar the
Fig. 4 Coronal and sagittal $\mathrm{CT}$ images of the talocalcaneal coalition in one patient
Fig. 5 a Pre-operative lateral radiographs of a female patient with a symptomatic talocalcaneal coalition. Note the presence of the C-sign in the hindfoot [7]. b Immediate postoperative radiograph. The gap of the posterior subtalar joint is closed. c Six weeks following surgery, bony fusion of the posterior subtalar arthrodesis is seen
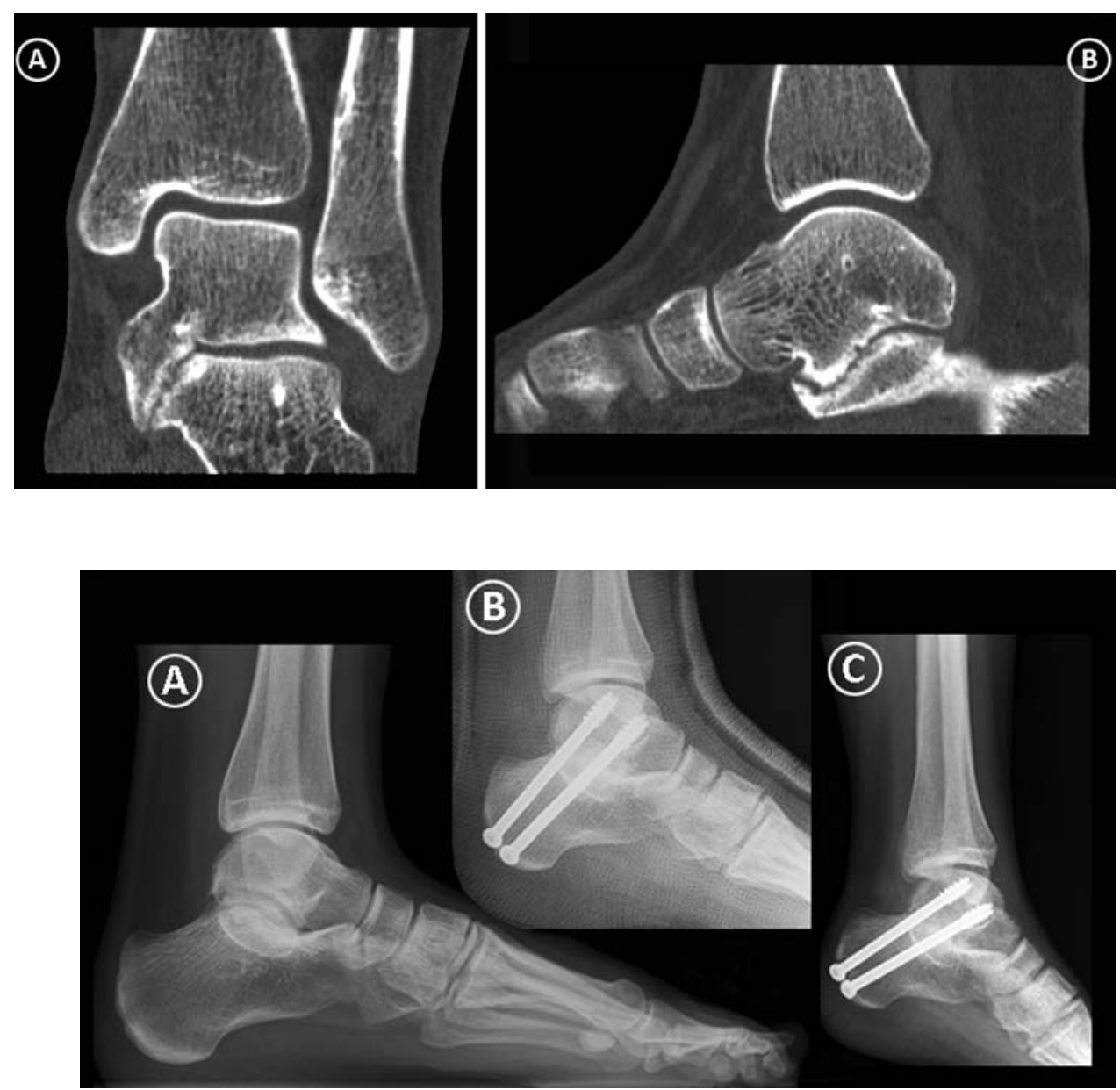
opening of the joint will always be limited. With the location of the sinus tarsi portal, the large blunt trocar is not interfering with the instruments and arthroscope that are in place via the posteromedial and posterolateral portals. A second posterolateral hindfoot portal was suggested for intra-articular placement of a large diameter blunt trocar for subtalar joint distraction [1, 4]. However, the accessory posterolateral portal is located close to the standard posterolateral portal, thereby reducing the working space in the hindfoot. Prone positioning of the patient allows for safe and easy placement of the two lag screws through the calcaneus and talus for fixation of the arthrodesis. In addition, the prone position facilitates accurate assessment of hindfoot alignment during surgery.

Lee et al. described a posterior arthroscopic approach with an accessory posterolateral portal for isolated subtalar arthrodesis with the patient in the prone position [4]. All 10 feet that were operated for painful isolated osteoarthritis of the subtalar joint achieved fusion within 10 weeks. In his series of 25 patients, Tasto reported an average time until complete fusion of 8.9 weeks (range, 6-16 weeks) for arthroscopic subtalar arthrodesis [11]. Perez Carro achieved radiographic union at a mean of 8 weeks (range, 6-11 weeks) in four patients [6]. With this 3-portal arthroscopic technique in these patients with a talocalcaneal coalition, radiographic bony fusion was seen in all three patients 6 weeks following surgery. No bone grafting was used. The studies available on arthroscopic subtalar arthrodesis and the use of bone grafting (medial tibial plateau bone marrow, cancellous allograft, synthetic bone graft) have not shown better results for the time to fusion of the arthrodesis or the fusion rate in comparison to studies not using bone grafting [1-3]. In our study, all debridement and removal of the cartilage of the posterior subtalar joint was done posterior to the interosseous ligament. This seems to become standard practice, as most studies showed that fusing solely the posterior facet in arthroscopic isolated subtalar arthrodesis is sufficient for bony fusion of the subtalar arthrodesis [1, 2, 4, 11]. With the 3-portal technique as described here, a safe and time-efficient arthroscopic subtalar arthrodesis can be performed even in cases with limited joint space such as in symptomatic talocalcaneal coalition.

Open Access This article is distributed under the terms of the Creative Commons Attribution Noncommercial License which permits any noncommercial use, distribution, and reproduction in any medium, provided the original author(s) and source are credited.

\section{References}

1. Amendola A, Lee KB, Saltzman CL, Suh JS (2007) Technique and early experience with posterior arthroscopic subtalar arthrodesis. Foot Ankle Int 28:298-302. doi:10.3113/FAI.2007. 0298

2. Boack DH, Manegold S, Friedebold A, Haas NP (2005) Arthroskopische in situ Arthrodese des Subtalar-Gelenkes. Orthopade 34:1245-1254. doi:10.1007/s00132-005-0903-9

3. Glanzmann MC, Sanhueza-Hernandez R (2007) Arthroscopic subtalar arthrodesis for symptomatic osteoarthritis of the hindfoot: a prospective study of 41 cases. Foot Ankle Int 28:2-7. doi: 10.3113/FAI.2007.0001

4. Lee KB, Saltzman CL, Suh JS, Wasserman L, Amendola A (2008) A posterior 3-portal arthroscopic approach for isolated subtalar arthrodesis. Arthroscopy 24:1306-1310. doi:10.1016/j. arthro.2006.01.025

5. Lemley F, Berlet G, Hill K, Philbin T, Isaac B, Lee T (2006) Current concepts review: tarsal coalition. Foot Ankle Int 27:1163-1169

6. Perez Carro L, Golanó P, Vega J (2007) Arthroscopic subtalar arthrodesis: the posterior approach in the prone position. Arthroscopy 23:445.e1-445.e4

7. Sakellariou A, Sallomi D, Janzen DL, Munk PL, Claridge RJ, Kiri VA (2000) Talocalcaneal coalition diagnosis with the C-sign on lateral radiographs of the ankle. J Bone Joint Surg Br 82:574578. doi:10.1302/0301-620X.82B4.10263

8. Scranton PE (1987) Treatment of symptomatic talocalcaneal coalition. J Bone Joint Surg Am 69:533-539

9. Swiontkowski MF, Scranton PE, Hansen S (1983) Tarsal coalitions: long-term results of surgical treatment. J Pediatr Orthop 3:287-292

10. Takakura Y, Sugimoto K, Tanaka Y, Tamai S (1991) Symptomatic talocalcaneal coalition. Its clinical significance and treatment. Clin Orthop Relat Res 269:249-256

11. Tasto JP (2003) Arthroscopic subtalar arthrodesis. Tech Foot Ankle Surg 2:122-128. doi:10.1097/00132587-200306000-00007

12. Van Dijk CN, Scholten PE, Krips R (2000) A 2-portal endoscopic approach for diagnosis and treatment of posterior ankle pathology. Arthroscopy 16:871-876. doi:10.1053/jars.2000.19430 